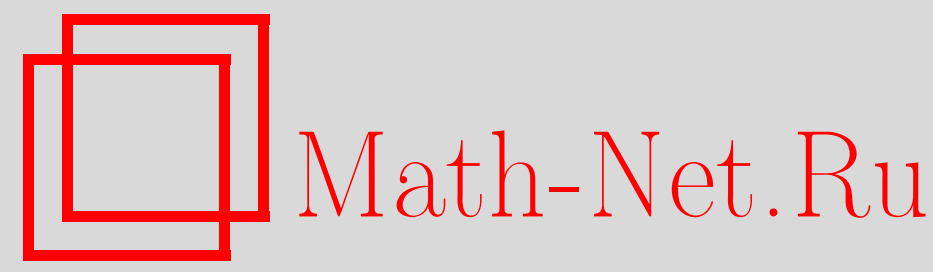

В. Н. Кокарев, Об уравнении несобственной выпуклой аффинной сферы: обобщение теоремы Ёргенса, Матем. сб., 2003, том 194, номер 11, 65-80

DOI: https://doi.org/10.4213/sm780

Использование Общероссийского математического портала Math-Net.Ru подразумевает, что вы прочитали и согласны с пользовательским соглашением http://www . mathnet.ru/rus/agreement

Параметры загрузки:

IP : 18.234 .197 .8

26 апреля 2023 г., 16:33:19 


\section{В.Н. Кокарев}

\section{Об уравнении несобственной выпуклой аффинной сферы: обобщение теоремы Ёргенса}

Доказьвается, что если функция $\varphi(t)$ положительной переменной $t$ принадлежит классу $C^{3, \alpha}$ и для достаточно малого положительного $\varepsilon\left(\varepsilon<10^{-4}\right)$ удовлетворяет условиям

$$
\begin{gathered}
1-\varepsilon \leqslant \varphi(t) \leqslant 1+\varepsilon, \quad t>0, \\
\left|\varphi^{\prime}(t)\right| \leqslant \varepsilon \frac{\varphi(t)}{t}, \quad t \geqslant 2 \sqrt{1-\varepsilon}, \\
\left|\varphi^{\prime \prime}(t)\right| \leqslant \varepsilon \frac{\varphi(t)}{t^{2}}, \quad t \geqslant 2 \sqrt{1-\varepsilon} \\
\left|\varphi^{\prime \prime \prime}(t)\right| \leqslant \varepsilon \frac{\varphi(t)}{t^{3}}, \quad t \geqslant 2 \sqrt{1-\varepsilon},
\end{gathered}
$$

то всякое полное решение $z(x, y)$ уравнения $z_{x x} z_{y y}-z_{x y}^{2}=\varphi\left(z_{x x}+z_{y y}\right)$ является квадратичным полиномом.

Библиограф̆ия: 8 названий.

\section{Введение}

Несобственная выпуклая аффинная сфера в подходящей системе координат задается в виде $z=z(x, y)$, где функция $z(x, y)$ удовлетворяет уравнению $z_{x x} z_{y y}-$ $z_{x y}^{2}=1$. В работе [1] K. Ёргенс доказал, что все полные решения этого уравнения являются квадратичными полиномами. Следовательно, все несобственные выпуклые аффинные сферы - эллиптические параболоиды, если в определение аффинной сферы включить требование полноты. Этот результат был обобщен по размерности Е. Калаби [2] и А.В. Погореловым [3].

Заметив, что $z_{x x} z_{y y}-z_{x y}^{2}=\lambda_{1} \lambda_{2}$, где $\lambda_{1}, \lambda_{2}$ - собственные значения гессиана функции $z(x, y)$, естественно поставить вопрос: насколько функция $\lambda_{1} \lambda_{2}$ важна для характеризации несобственных выпуклых аффинных сфер? А именно, что можно сказать о полной поверхности, заданной в виде $z=z(x, y)$, если собственные значения $\lambda_{1}, \lambda_{2}$ гессиана функции $z(x, y)$ удовлетворяют уравнению $f\left(\lambda_{1}, \lambda_{2}\right)=0$, а кривая, заданная этим уравнением в плоскости переменных $\lambda_{1}, \lambda_{2}$, достаточно близка к гиперболе $\lambda_{1} \lambda_{2}-1=0$ ? Точное описание этой близости дадим ниже, а пока пусть $f\left(\lambda_{1}, \lambda_{2}\right)$ - регулярная, симметрическая функция положительных переменных такая, что $f_{\lambda_{1}}^{2}+f_{\lambda_{2}}^{2} \neq 0$, кривая, заданная уравнением $f\left(\lambda_{1}, \lambda_{2}\right)=0$, может касаться прямых $\lambda_{1}+\lambda_{2}=c$ только при $\lambda_{1}=\lambda_{2}$ и кривизна этой кривой

Работа вьполнена при поддержке Российского фонда фундаментальных исследований (грант № 02-01-00017). 
при $\lambda_{1}=\lambda_{2}$ не равна нулю. Введя функции $\sigma_{1}=\lambda_{1}+\lambda_{2}$ и $\sigma_{2}=\lambda_{1} \lambda_{2}$, получим $f\left(\lambda_{1}, \lambda_{2}\right)=F\left(\sigma_{1}, \sigma_{2}\right)$. При $\lambda_{1} \neq \lambda_{2}$ находим

$$
\frac{\partial F}{\partial \sigma_{2}}=\frac{f_{\lambda_{2}}-f_{\lambda_{1}}}{\lambda_{1}-\lambda_{2}} \neq 0
$$

Если функция $f$ имеет класс $C^{2}$, то при $\lambda_{1}=\lambda_{2}$ производная $\partial F / \partial \sigma_{2}=f_{\lambda_{1} \lambda_{2}}-$ $f_{\lambda_{1} \lambda_{1}}$. Так как при $\lambda_{1}=\lambda_{2}$ имеем $f_{\lambda_{1}}=f_{\lambda_{2}}, f_{\lambda_{1} \lambda_{1}}=f_{\lambda_{2} \lambda_{2}}$, то $\left.\left(\partial F / \partial \sigma_{2}\right)\right|_{\lambda_{1}=\lambda_{2}}$ пропорциональна $f_{\lambda_{1} \lambda_{1}} f_{\lambda_{2}}^{2}-2 f_{\lambda_{1} \lambda_{2}} f_{\lambda_{1}} f_{\lambda_{2}}+f_{\lambda_{2} \lambda_{2}} f_{\lambda_{1}}^{2}$ и в силу необрашения в нуль кривизны при $\lambda_{1}=\lambda_{2}$ производная $\partial F / \partial \sigma_{2}$ и здесь существует и не обращается в нуль.

Тогда уравнение $F\left(\sigma_{1}, \sigma_{2}\right)=0$ можно разрешить относительно $\sigma_{2}$ :

$$
\sigma_{2}=\varphi\left(\sigma_{1}\right) .
$$

В таком виде мы и будем рассматривать соотношение между $\lambda_{1}$ и $\lambda_{2}$, наложив на функцию $\varphi$ некоторые ограничения.

\section{§1. Постановка и обсуждение задачи}

Нам будет удобно несколько обобщить понятие собственного значения гессиана функции, являющейся графиком поверхности. Пусть полная поверхность $\Phi$ и эллиптический параболоид П заданы в прямоугольной системе координат $x^{1}, x^{2}, x^{3}=z$ уравнениями $z=z\left(x^{1}, x^{2}\right)$ и $z=z^{0}\left(x^{1}, x^{2}\right)=\frac{1}{2} c_{i j} x^{i} x^{j}$ соответственно. При суммировании индексы всюду пробегают значения 1,2 . Обозначим $\partial z / \partial x^{i}=z_{i}, \partial^{2} z / \partial x^{i} \partial x^{j}=z_{i j}$ и т.д. Через $\lambda_{1}, \lambda_{2}$ обозначим экстремумы формы $z_{i j} \xi^{i} \xi^{j}$ относительно формы $z_{i j}^{0} \xi^{i} \xi^{j}=c_{i j} \xi^{i} \xi^{j}$. Они являются корнями уравнения

$$
\operatorname{det}\left(z_{i j}-\lambda c_{i j}\right)=0 .
$$

Пусть поверхность $\Phi$ такова, что $\lambda_{1}, \lambda_{2}$ удовлетворяют уравнению (1), где функция $\varphi(t)$ определена для $t>0$, имеет класс $C^{3, \alpha}$ и для нее выполняются следующие условия:

$$
\begin{gathered}
1-\varepsilon \leqslant \varphi(t) \leqslant 1+\varepsilon \\
\left|\varphi^{\prime}(t)\right| \leqslant \varepsilon \frac{\varphi(t)}{t}, \\
\left|\varphi^{\prime \prime}(t)\right| \leqslant \varepsilon \frac{\varphi(t)}{t^{2}} \\
\left|\varphi^{\prime \prime \prime}(t)\right| \leqslant \varepsilon \frac{\varphi(t)}{t^{3}}
\end{gathered}
$$

с положительным $\varepsilon$. Что тогда можно сказать о поверхности $\Phi$ ? Мы доказываем, что если $\varepsilon$ достаточно мало, то поверхность $\Phi$ будет эллиптическим параболоидом.

Если выполняются условия (3)-(5) с $\varepsilon<1$, то функция $\ln \sigma_{2}-\ln \varphi\left(\sigma_{1}\right)$ при $\lambda_{1}>0, \lambda_{2}>0$ и область $\left(x^{1}\right)^{2}+\left(x^{2}\right)^{2} \leqslant 1$ удовлетворяют всем условиям теоремњ 2 из [4]. Поэтому задача Дирихле для уравнения $\ln \sigma_{2}-\ln \varphi\left(\sigma_{1}\right)=0$ разрешима в круге $\left(x^{1}\right)^{2}+\left(x^{2}\right)^{2} \leqslant 1$ для любого регулярного граничного условия. Значит, уравнение (1) при ограничениях (3)-(5) имеет много локальных решений. 
Из наших результатов будет следовать, что те из них, которые не являются квадратичными полиномами, не могут быть продолжены до полных, по крайней мере, для достаточно малых $\varepsilon$.

По теореме Виета для $\sigma_{1}=\lambda_{1}+\lambda_{2}$ и $\sigma_{2}=\lambda_{1} \lambda_{2}$ получаем

$$
\sigma_{1}=\frac{c_{11} z_{22}+2 c_{12} z_{12}+c_{22} z_{11}}{\operatorname{det}\left(c_{i j}\right)}=\widetilde{c}^{i j} z_{i j}, \quad \sigma_{2}=\frac{\operatorname{det}\left(z_{i j}\right)}{\operatorname{det}\left(c_{i j}\right)}
$$

где $\widetilde{c}^{i j}$ - элементы матрицы, обратной к матрице из коэффициентов $c_{i j}$. Из уравнения (1) получаем $\operatorname{det}\left(z_{i j}\right)=\operatorname{det}\left(c_{i j}\right) \varphi\left(\widetilde{c}^{i j} z_{i j}\right)$. Уравнение (2) для нахождения $\lambda_{1}, \lambda_{2}$ инвариантно при афффинных преобразованиях координат $x^{1}, x^{2}$. Значит, если афффинным преобразованием вида $\left(x^{1}, x^{2}, z\right) \mapsto\left(\alpha_{i}^{1} x^{i}, \alpha_{i}^{2} x^{i}, z\right)$ одновременно перевести поверхности $\Phi$ и П в поверхности $\Phi^{\prime}$ и $\Pi^{\prime}$, то $\lambda_{1}, \lambda_{2}$ в соответствующих точках поверхностей $\Phi$ и $\Phi^{\prime}$, вычисленные по поверхностям $\Phi, \Pi$ и $\Phi^{\prime}, \Pi^{\prime}$, будут одинаковьми. Чтобы не усложнять вычисления, будем считать, что $\operatorname{det}\left(c_{i j}\right)=1$, и будем допускать только аффинные преобразования, coxраняющие $\operatorname{det}\left(c_{i j}\right)$. Тогда для поверхности $\Phi$ получаем уравнение

$$
\operatorname{det}\left(z_{i j}\right)=\varphi\left(c_{11} z_{22}+2 c_{12} z_{12}+c_{22} z_{11}\right)
$$

Может возникнуть вопрос: зачем нужно рассматривать уравнение вида (7), если унимодулярным аффинным преобразованием поверхностей можно добиться того, что $c_{i j}=\delta_{i j}$ ? Дело в том, что нам придется впоследствии подвергать поверхности аффинным преобразованиям, при которых уравнение с $c_{i j}=\delta_{i j}$ все равно приобретет вид (7). Поэтому нам нужно получить информацию о решениях этого уравнения. Из (3) следует, что все решения уравнения (7) строго вьпуклые, а поверхности $\Phi$ и П расположены вогнутостью в одну сторону (положим вверх).

Приступим к исследованию полных решений уравнения (7) при условиях (3)-(6).

\section{§ 2. $C^{2}$-оценка решения уравнения $(7)$ в ограниченной области}

Так как к функции $z\left(x^{1}, x^{2}\right)$ можно прибавлять слагаемые вида $c_{1} x^{1}+c_{2} x^{2}+$ $c$, то без ограничения общности считаем, что прямоугольная система координат $x^{1}, x^{2}, x^{3}=z$ такова, что поверхность $\Phi$ касается плоскости $z=0$. Через $G_{h}$ обозначим область на плоскости $z=0$, где $z\left(x_{1}, x_{2}\right) \leqslant h$, и пусть $d_{h}-$ диаметр этой области. Оценим производные $z_{i}$ в области $G_{1}$. Для случая $\varphi(t)=1$ оценка дана в [3].

Возьмем точку $\left(x^{1}, x^{2}\right) \in G_{1}$ и построим конус $V$ с вершиной в точке $S\left(x^{1}, x^{2}\right.$, $\left.z\left(x^{1}, x^{2}\right)\right)$, который проектирует пересечение плоскости $z=2$ с поверхностью $\Phi$. Через $\Phi_{h}$ обозначим часть поверхности $\Phi$, для которой $z\left(x^{1}, x^{2}\right) \leqslant h$. Пусть $V^{*}$ и $\Phi_{2}^{*}$ - нормальные изображения $V$ и $\Phi_{2}$ соответственно (см. $[3 ; \S 5$, п. 1]) на плоскости $p_{1}, p_{2}$. В силу выпуклости $\Phi_{2}$ имеем $V^{*} \subset \Phi_{2}^{*}$, следовательно, для площадей получается неравенство $\sigma\left(V^{*}\right) \leqslant \sigma\left(\Phi_{2}^{*}\right)$. Так как высота конуса $V$ не менее 1 , а диаметр основания равен $d_{2}$, то круг $p_{1}^{2}+p_{2}^{2} \leqslant 1 / d_{2}^{2}$ принадлежит $V^{*}$. Точка $\left(p_{1}, p_{2}\right)=\left(z_{1}\left(x^{1}, x^{2}\right), z_{2}\left(x^{1}, x^{2}\right)\right)$ тоже принадлежит $V^{*}$. Значит, в $V^{*}$ лежит треугольник с основанием $2 / d_{2}$, высотой $\left|z_{i}\left(x^{1}, x^{2}\right)\right|$, площадью $\left|z_{i}\left(x^{1}, x^{2}\right)\right| / d_{2}$. Значит, $\sigma\left(V^{*}\right) \geqslant\left|z_{i}\left(x^{1}, x^{2}\right)\right| / d_{2}$. 
Далее,

$$
\begin{aligned}
\sigma\left(\Phi_{2}^{*}\right) & =\int_{\Phi_{2}^{*}} d p_{1} d p_{2}=\int_{G_{2}} \frac{\partial\left(p_{1}, p_{2}\right)}{\partial\left(x^{1}, x^{2}\right)} d x^{1} d x^{2}=\int_{G_{2}} \operatorname{det}\left(z_{i j}\right) d x^{1} d x^{2} \\
& =\int_{G_{2}} \varphi d x^{1} d x^{2} \leqslant \frac{1}{4}(1+\varepsilon) \pi d_{2}^{2} .
\end{aligned}
$$

Из $\sigma\left(V^{*}\right) \leqslant \sigma\left(\Phi_{2}^{*}\right)$ получаем оценку сверху на $\left|z_{i}\left(x^{1}, x^{2}\right)\right|$ для всех точек области $G_{1}$. Оценка зависит от $\varepsilon$ и $d_{2}$ - диаметра сечения поверхности $\Phi$ на высоте 2 .

Теперь оценим вторые производные решения уравнения (7) в области $G_{1 / 2}$. Модифицируя прием А.В. Погорелова (см. [5; $§ 5$, п. 2, док-во теоремы 5.1]), введем в $G_{1}$ функцию точки и направления

$$
w=(1-z)^{k} e^{\left(z_{1}^{2}+z_{2}^{2}\right) / 2} z_{\alpha \alpha}
$$

где $\alpha$ означает дифференцирование в некотором направлении, а постоянная $k>0$ будет выбрана позже. Функция $w$ достигает максимума, равного $w_{0}$, во внутренней точке $O$ области $G_{1}$ для некоторого направления $\alpha_{0}$. Это направление является главным направлением в точке $O$ квадратичной формы $d^{2} z$. Повернем оси $x^{1}, x^{2}$ так, чтобы направление оси $x^{1}$ совпало с направлением $\alpha_{0}$, тогда $z_{12}=0$ в точке $O$. В новых координатах уравнение поверхности $\Phi$ будет по-прежнему иметь вид (7), но, вообще говоря, с другими $c_{i j}$. Оставим за этими коэффициентами прежние обозначения. Опять $\operatorname{det}\left(c_{i j}\right)=1$.

Продифференцировав (7) в точке $O$ по $x^{i}$ и дважды по $x^{1}$, получим после преобразований

$$
\begin{gathered}
\frac{z_{11 i}}{z_{11}}+\frac{z_{22 i}}{z_{22}}=\left(c_{22} z_{11 i}+2 c_{12} z_{12 i}+c_{11} z_{22 i}\right) \frac{\varphi^{\prime}}{\varphi}, \\
\frac{z_{22}-\varphi^{\prime} c_{22}}{z_{11} z_{22}} z_{1111}-2 \frac{\varphi^{\prime} c_{12}}{z_{11} z_{22}} z_{1112}+\frac{z_{11}-\varphi^{\prime} c_{11}}{z_{11} z_{22}} z_{1122}+2 \frac{z_{111} z_{221}-z_{112}^{2}}{z_{11} z_{22}} \\
=\left(c_{22} z_{111}+2 c_{12} z_{112}+c_{11} z_{221}\right)^{2} \frac{\varphi^{\prime \prime}}{\varphi} .
\end{gathered}
$$

Так как функция $w$ в точке $O$ достигает максимума, то в этой точке $d(\ln w)=0$, $d^{2}(\ln w) \leqslant 0$. Вычислив, получим

$$
\begin{gathered}
(\ln w)_{i}=\frac{z_{11 i}}{z_{11}}+z_{i} z_{i i}+\frac{k z_{i}}{z-1}=0 \\
(\ln w)_{i j}=\frac{z_{11 i j}}{z_{11}}-\frac{z_{11 i} z_{11 j}}{z_{11}^{2}}-\frac{k z_{i} z_{j}}{(z-1)^{2}}+\frac{k z_{i j}}{z-1}+z_{1 i} z_{1 j}+z_{2 i} z_{2 j}+z_{1} z_{1 i j}+z_{2} z_{2 i j}
\end{gathered}
$$

Из условия (4) получаем

$$
\left|\varphi^{\prime} c_{11}\right| \leqslant \frac{\varepsilon \varphi c_{11}}{c_{11} z_{22}+c_{22} z_{11}} \leqslant \frac{\varepsilon z_{11} z_{22} c_{11}}{c_{11} z_{22}}=\varepsilon z_{11}
$$


Аналогично, $\left|\varphi^{\prime} c_{22}\right| \leqslant \varepsilon z_{22}$. Поэтому при $\varepsilon<1$ получаем, что

$$
\operatorname{det}\left(\begin{array}{cc}
z_{11}-\varphi^{\prime} c_{11} & -\varphi^{\prime} c_{12} \\
-\varphi^{\prime} c_{12} & z_{22}-\varphi^{\prime} c_{22}
\end{array}\right)>0
$$

Следовательно, форма

$$
\psi=\frac{1}{z_{22}}\left(\left(z_{11}-\varphi^{\prime} c_{11}\right) \xi_{1}^{2}-2 \varphi^{\prime} c_{12} \xi_{1} \xi_{2}+\left(z_{22}-\varphi^{\prime} c_{22}\right) \xi_{2}^{2}\right)>0
$$

Тогда для следа форм $\psi$ и $d^{2}(\ln w)$ получаем

$$
\begin{aligned}
& \frac{z_{22}-\varphi^{\prime} c_{22}}{z_{11} z_{22}} z_{1111}-2 \frac{\varphi^{\prime} c_{12}}{z_{11} z_{22}} z_{1112}+\frac{z_{11}-\varphi^{\prime} c_{11}}{z_{11} z_{22}} z_{1122}-\frac{z_{22}-\varphi^{\prime} c_{22}}{z_{11}^{2} z_{22}} z_{111}^{2} \\
& +2 \frac{\varphi^{\prime} c_{12}}{z_{11}^{2} z_{22}} z_{111} z_{112}-\frac{z_{11}-\varphi^{\prime} c_{11}}{z_{11}^{2} z_{22}} z_{112}^{2}-\frac{\left(z_{22}-\varphi^{\prime} c_{22}\right) k z_{1}^{2}}{z_{22}(z-1)^{2}}+2 \frac{\varphi^{\prime} c_{12} k z_{1} z_{2}}{z_{22}(z-1)^{2}} \\
& \quad-\frac{\left(z_{11}-\varphi^{\prime} c_{11}\right) k z_{2}^{2}}{z_{22}(z-1)^{2}}+\frac{\left(z_{22}-\varphi^{\prime} c_{22}\right) k z_{11}}{z_{22}(z-1)}+\frac{\left(z_{11}-\varphi^{\prime} c_{11}\right) k}{z-1} \\
& +\frac{\left(z_{22}-\varphi^{\prime} c_{22}\right)\left(z_{11}^{2}+z_{1} z_{111}+z_{2} z_{112}\right)}{z_{22}}-2 \frac{\varphi^{\prime} c_{12}\left(z_{1} z_{112}+z_{2} z_{122}\right)}{z_{22}} \\
& +\frac{\left(z_{11}-\varphi^{\prime} c_{11}\right)\left(z_{22}^{2}+z_{1} z_{122}+z_{2} z_{222}\right)}{z_{22}} \leqslant 0 .
\end{aligned}
$$

Вычтем из (13) почленно (9) и подставим в получившееся неравенство $z_{222}$ и $z_{221}$, выразив их из (8) при $i=1$ и $i=2$. Получим

$$
\begin{aligned}
& \frac{\left(z_{22}-\varphi^{\prime} c_{22}\right)\left(z_{11}+\varphi^{\prime} c_{11}\right)}{\left(z_{11}-\varphi^{\prime} c_{11}\right) z_{11}^{2} z_{22}} z_{111}^{2}-2 \frac{\varphi^{\prime} c_{12}\left(z_{11}+\varphi^{\prime} c_{11}\right)}{\left(z_{11}-\varphi^{\prime} c_{11}\right) z_{11}^{2} z_{22}} z_{111} z_{112}+\frac{z_{11}+\varphi^{\prime} c_{11}}{z_{11}^{2} z_{22}} z_{112}^{2} \\
& -\frac{\left(z_{22}-\varphi^{\prime} c_{22}\right) k z_{1}^{2}}{z_{22}(z-1)^{2}}+2 \frac{\varphi^{\prime} c_{12} k z_{1} z_{2}}{z_{22}(z-1)^{2}}-\frac{\left(z_{11}-\varphi^{\prime} c_{11}\right) k z_{2}^{2}}{z_{22}(z-1)^{2}} \\
& \quad+\frac{\left(z_{22}-\varphi^{\prime} c_{22}\right) k z_{11}}{z_{22}(z-1)}+\frac{\left(z_{11}-\varphi^{\prime} c_{11}\right) k}{z-1}+\frac{\left(z_{22}-\varphi^{\prime} c_{22}\right) z_{11}^{2}}{z_{22}} \\
& +\left(z_{11}-\varphi^{\prime} c_{11}\right) z_{22}+\frac{\varphi^{\prime \prime}}{\varphi}\left(\frac{c_{22} z_{11}-c_{11} z_{22}}{z_{11}-\varphi^{\prime} c_{11}} z_{111}+2 \frac{c_{12} z_{11}}{z_{11}-\varphi^{\prime} c_{11}} z_{112}\right)^{2} \leqslant 0 .
\end{aligned}
$$

С помощью (10) получаем неравенство

$$
\frac{k z_{2}^{2}}{(z-1)^{2}} \leqslant \frac{2 z_{112}^{2}}{k z_{11}^{2}}+\frac{2 z_{2}^{2} z_{22}^{2}}{k} .
$$

Так как

$$
\begin{aligned}
& \left(c_{22} z_{11}-c_{11} z_{22}\right)^{2} \leqslant\left(c_{22} z_{11}+c_{11} z_{22}\right)^{2}, \\
& \left(c_{22} z_{11}+c_{11} z_{22}\right)^{2} \geqslant 4 c_{11} c_{22} z_{11} z_{22} \geqslant 4 c_{12}^{2} z_{11} z_{22},
\end{aligned}
$$


то с учетом (5) получаем

$$
\begin{aligned}
& \frac{\varphi^{\prime \prime}}{\varphi}\left(\frac{c_{22} z_{11}-c_{11} z_{22}}{z_{11}-\varphi^{\prime} c_{11}} z_{111}+\frac{2 c_{12} z_{11}}{z_{11}-\varphi^{\prime} c_{11}} z_{112}\right)^{2} \\
& \quad \geqslant-\frac{2 \varepsilon}{\left(z_{11}-\varphi^{\prime} c_{11}\right)^{2}} z_{111}^{2}-\frac{2 \varepsilon z_{11}}{z_{22}\left(z_{11}-\varphi^{\prime} c_{11}\right)^{2}} z_{112}^{2} .
\end{aligned}
$$

Так как $\left|\varphi^{\prime} c_{i i}\right| \leqslant \varepsilon z_{i i}$, то при $(1-\varepsilon)^{3}>4 \varepsilon(\varepsilon<\approx 0.15)$ сушествует такое $k>0$, зависящее только от $\varepsilon$ (мы еще обязательно возьмем $k \geqslant 2$ ), что квадратичная форма относительно $z_{111}, z_{112}$ неотрицательна:

$$
\begin{aligned}
& \left(\frac{\left(z_{22}-\varphi^{\prime} c_{22}\right)\left(z_{11}+\varphi^{\prime} c_{11}\right)}{z_{11}^{2} z_{22}\left(z_{11}-\varphi^{\prime} c_{11}\right)}-\frac{2 \varepsilon}{\left(z_{11}-\varphi^{\prime} c_{11}\right)^{2}}\right) z_{111}^{2}-\frac{2 \varphi^{\prime} c_{12}\left(z_{11}+\varphi^{\prime} c_{11}\right)}{z_{11}^{2} z_{22}\left(z_{11}-\varphi^{\prime} c_{11}\right)} z_{111} z_{112} \\
& +\left(\frac{z_{11}+\varphi^{\prime} c_{11}}{z_{11}^{2} z_{22}}-\frac{2\left(z_{11}-\varphi^{\prime} c_{11}\right)}{k z_{11}^{2} z_{22}}-\frac{2 \varepsilon z_{11}}{z_{22}\left(z_{11}-\varphi^{\prime} c_{11}\right)^{2}}\right) z_{112}^{2} \geqslant 0 .
\end{aligned}
$$

С помощью этого неравенства из (14)-(16) с учетом (3) и того, что

$$
z_{11} z_{22}=\varphi, \quad\left|\frac{\varphi^{\prime} c_{12}}{z_{22}}\right| \leqslant \frac{\varepsilon \varphi\left|c_{12}\right|}{z_{22}\left(c_{11} z_{22}+c_{22} z_{11}\right)} \leqslant \frac{\varepsilon \varphi\left|c_{12}\right|}{2 z_{22} \sqrt{c_{11} c_{22} z_{11} z_{22}}} \leqslant \frac{\varepsilon z_{11}}{2 \sqrt{1-\varepsilon}},
$$

получим

$$
\begin{aligned}
& -\frac{(1+\varepsilon) k z_{1}^{2}}{(z-1)^{2}}-\frac{\varepsilon k\left|z_{1} z_{2}\right| z_{11}}{\sqrt{1-\varepsilon}(z-1)^{2}}-\frac{2(1+\varepsilon)^{2} z_{2}^{2}}{k}+\frac{2(1+\varepsilon) k z_{11}}{z-1} \\
& \quad+(1-\varepsilon) z_{11}^{2}+(1-\varepsilon)^{2} \leqslant 0
\end{aligned}
$$

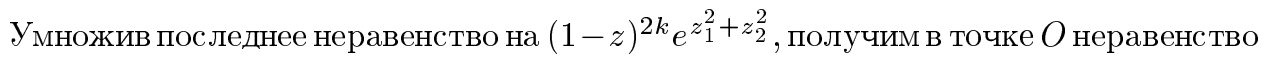
вида

$$
(1-\varepsilon) w_{0}^{2}+Q w_{0}+R \leqslant 0,
$$

где $Q$ и $R$ содержат $(1-z)$ в неотрицательных степенях.

Так как в области $G_{1} 0 \leqslant 1-z \leqslant 1$, то коэффициенты $Q$ и $R$ ограничены в зависимости от $\varepsilon$ и первых производных функции $z$ в $G_{1}$. Из (17) получаем оценку на $w_{0}$, которая при фиксированном $\varepsilon$ зависит в конечном счете только от $d_{2}$. Обозначив ее через $w_{0}\left(d_{2}\right)$, получаем для всех точек и всех направлений в области $G_{1}$

$$
(1-z)^{k} e^{\left(z_{1}^{2}+z_{2}^{2}\right) / 2} z_{\alpha \alpha} \leqslant w_{0}\left(d_{2}\right)
$$

Отсюда для всех точек и всех направлений в области $G_{1 / 2}$

$$
z_{\alpha \alpha} \leqslant 2^{k} w_{0}\left(d_{2}\right)
$$

Постоянная $k$ выбирается в зависимости только от $\varepsilon$.

Может вызвать удивление то, что оценка не зависит от коэффициентов $c_{i j}$. Это не значит, конечно, что поверхность П не играет никакой роли. Например, для $\Pi=\Phi: z=\frac{1}{2} c_{i j} x^{i} x^{j}$ как раз $z_{i i}=c_{i i}$. Утверждается лишш, что оценка может быть выражена только через $d_{2}$ (которое, вообще говоря, зависит и от коэффициентов $c_{i j}$ ). 


\section{§3. Глобальная оценка для вторых производных решения уравнения $(7)$}

Здесь мы используем несколько модифиццированньй метод из [3].

Через $T_{h}$ обозначим пересечение плоскости $z=h$ с выпуклым телом, ограниченным поверхностью $\Phi$. Не ограничивая общности, считаем, что плоскость $z=0$ касается поверхности $\Phi$ в точке $S$, а ось $z$ проходит через центр тяжести сечения $T_{h}$. Пусть $p$ - параллелограмм минимальной площади, содержащий $T_{h}$, центр которого совпадает с центром тяжести $T_{h}$. Подвергнем поверхности $\Phi$ и П унимодулярному аффинному преобразованию $\chi$ вида $\left(x^{1}, x^{2}, z\right) \mapsto\left(\alpha_{i}^{1} x^{i}, \alpha_{i}^{2} x^{i}, z\right)$, переводящему параллелограмм $p$ в квадрат $q$ со стороной $b$, а поверхности $\Phi$ и $\Pi-$ в поверхности $\Phi^{\prime}$ и $\Pi^{\prime}$. Оси $x^{1}, x^{2}$ можно выбрать так, что это преобразование будет иметь вид $\chi:\left(x^{1}, x^{2}, z\right) \mapsto\left(\mu_{1} x^{1}, \mu_{2} x^{2}, z\right)$. При этом поверхности $\Phi^{\prime}, \Pi^{\prime}$ удовлетворяют уравнению (7) с той же функцией $\varphi$ и $\operatorname{det} c_{i j}=1$. Докажем, что величина $h / b^{2}$ ограничена сверху и снизу некоторыми положительньми константами, завислщими только от $\varepsilon$.

Для поверхности $\Phi^{\prime}$ введем $\Phi_{h}^{\prime}, G_{h}^{\prime}, \Phi_{h}^{\prime *}$ так же, как вводились $\Phi_{h}, G_{h}, \Phi_{h}^{*}$ для поверхности $\Phi$ в $\S 2$.

Тогда, как и в $§ 2$,

$$
\sigma\left(\Phi_{h}^{\prime *}\right)=\int_{\Phi_{h}^{\prime *}} d p_{1} d p_{2}=\int_{G_{h}^{\prime}} \operatorname{det}\left(z_{i j}\right) d x^{1} d x^{2}=\int_{G_{h}^{\prime}} \varphi d x^{1} d x^{2} \leqslant(1+\varepsilon) b^{2} .
$$

Площадь нормального изображения конуса, проектирующего квадрат $q$ из точки $S^{\prime}=\chi(S)$, не менее чем $2 h^{2} / b^{2}$. Сравнивая ее с $\sigma\left(\Phi_{h}^{\prime}{ }^{*}\right)$, получаем $2 h^{2} / b^{2} \leqslant(1+\varepsilon) b^{2}$ или $h / b^{2} \leqslant \sqrt{(1+\varepsilon) / 2}$.

Подвергнем квадрат $q$ гомотетии с коэффициентом $k=\frac{1}{3}$ относительно его центра. Известно, что полученный квадрат $q^{\prime}$ будет содержаться в $T_{h}^{\prime}=\chi\left(T_{h}\right)$. Спроектируем квадрат $q^{\prime}$ конусом $V$ из точки $(0,0,-h)$. Часть поверхности $\Phi_{h}^{\prime}$, лежащую внутри этого конуса, обозначим $\Phi_{V}$. Обозначим нормальные изображения $\Phi_{V}$ и $V$ через $\Phi_{V}^{*}$ и $V^{*}$. Для них имеем $\Phi_{V}^{*} \subset V^{*}$. Для площади $V^{*}$ сразу получаем $\sigma\left(V^{*}\right)=288 h^{2} / b^{2}$. Далее, спроектируем квадрат $q^{\prime}$ на плоскость $z=0$, а затем подвергнем гомотетии с коэффициентом $\frac{1}{2}$ относительно начала координат. Полученный квадрат обозначим $\frac{1}{2} \operatorname{pr}\left(q^{\prime}\right)$. Тогда

$$
\sigma\left(\Phi_{V}^{*}\right) \geqslant \int_{(1 / 2) \operatorname{pr}\left(q^{\prime}\right)} \operatorname{det}\left(z_{i j}\right) d x^{1} d x^{2}=\int_{(1 / 2) \operatorname{pr}\left(q^{\prime}\right)} \varphi d x^{1} d x^{2} \geqslant(1-\varepsilon) \frac{b^{2}}{36} .
$$

Из $\sigma\left(\Phi_{V}^{*}\right) \leqslant \sigma\left(V^{*}\right)$ получаем оценку снизу на $h / b^{2}: h / b^{2} \geqslant \sqrt{1-\varepsilon} /(72 \sqrt{2})$.

Подвергнем поверхности $\Phi^{\prime}$ и $\Pi^{\prime}$ афффинному преобразованию $\nu:\left(x^{1}, x^{2}, z\right) \mapsto$ $\left(x^{1} \sqrt{2} / \sqrt{h}, x^{2} \sqrt{2} / \sqrt{h}, 2 z / h\right)$. В результате получим поверхности $\bar{\Phi}$ и $\bar{\Pi}=\Pi^{\prime}$, при этом поверхность $\bar{\Phi}$ будет удовлетворять уравнению $(7)$ и $\operatorname{det}\left(c_{i j}\right)=1$. В результате композиции $\nu \circ \chi$ наших двух аффинных преобразований плоскость $z=h$ перейдет в плоскость $z=2$, при этом сечение поверхности $\bar{\Phi}$ плоскостью $z=2$ будет содержаться в квадрате со стороной $b \sqrt{2} / h$. Из оценки снизу на $h / b^{2}$ получаем, что диаметр этого сечения $\bar{d}_{2}$ ограничен сверху в зависимости только от $\varepsilon$. 
Пусть поверхность $\bar{\Phi}$ задается графиком функции $\bar{z}\left(x^{1}, x^{2}\right)$. В соответствующих точках поверхностей $\Phi$ и $\bar{\Phi}$ для вторых производных функций $z\left(x^{1}, x^{2}\right)$ и $\bar{z}\left(x^{1}, x^{2}\right)$ имеет место соотношение

$$
\mu_{\alpha}^{2} \bar{z}_{\alpha \alpha}=z_{\alpha \alpha}
$$

Запишем это соотношение для точки $S$ на поверхности $\Phi$ и соответствуюшей ей точки $\bar{S}$ на поверхности $\bar{\Phi}$

$$
\mu_{\alpha}^{2} \bar{z}_{\alpha \alpha}(\bar{S})=z_{\alpha \alpha}(S)
$$

Из $\S 2$ получаем оценки сверху на $\bar{z}_{\alpha \alpha}(\bar{S})$ через $\bar{d}_{2}, \varepsilon$ и на $z_{\alpha \alpha}(S)$ через $d_{2}$, $\varepsilon$. Так как обе поверхности $\bar{\Phi}$ и $\Phi$ удовлетворяют уравнению $(7)$, то с учетом условий $(3)$ получаем на $\bar{z}_{\alpha \alpha}(\bar{S})$ и $z_{\alpha \alpha}(S)$ положительные оценки снизу. Следовательно, коэффициенты $\mu_{\alpha}$ оцениваются сверху и снизу через $d_{2}$ и $\varepsilon$.

Множество точек плоскости $z=0$, для которых $\bar{z}\left(x^{1}, x^{2}\right) \leqslant \frac{1}{2}$, обозначим через $\bar{G}_{1 / 2}$. Из результатов $\S 2$ следует существование оценки, зависящей от $\bar{d}_{2}$ (значит, только от $\varepsilon)$, на $\bar{z}_{\alpha \alpha}$ в области $\bar{G}_{1 / 2}$. Эта область является образом области $\bar{G}_{h / 4}$ при нашем аффинном преобразовании $\nu \circ \chi$. Возврашаясь к соотношению (18), получаем, что для вторых производных $z_{\alpha \alpha}$ решения $z\left(x^{1}, x^{2}\right)$ уравнения (7) всюду в области $G_{h / 4}$ сушествует оценка сверху. При фиксированном $\varepsilon$ она, в конечном счете, может быть выражена только через $d_{2}$. Так как $h>0$ произвольно, то оценка имеет место для всех точек поверхности $\Phi$. Из уравнения (7) с учетом условия (3) получается также положительная оценка на $z_{\alpha \alpha}$ снизу.

В силу полноты поверхности $\Phi$ и ограниченности $z_{\alpha \alpha}$ сверху мы получаем, что проекцией поверхности $\Phi$ является вся плоскость $z=0$. Это делает возможным введение в следуюшем параграфе на всей плоскости $z=0$ специальной метрики.

\section{§4. Дифференциальное неравенство для решения уравнения (7)}

Запишем уравнение $(7)$ в виде $\operatorname{det}\left(z_{i j}\right)-\varphi\left(c_{11} z_{22}+2 c_{12} z_{12}+c_{22} z_{11}\right)=0$. Рассматривая левую часть как функцию от производных $z_{i j}$, обозначим $\operatorname{det}\left(z_{i j}\right)-$ $\varphi\left(c_{11} z_{22}+2 c_{12} z_{12}+c_{22} z_{11}\right)=\Omega\left(z_{i j}\right)$. Если допускать только афффинные преобразования переменных $x^{1}, x^{2}$, то производные $\partial \Omega / \partial z_{i j}$ являются координатами симметричного тензора типа $(0,2)$. Введем метрику на плоскости $z=0$, выбрав $g^{i j}=\partial \Omega / \partial z_{i j}$ в качестве координат контравариантного метрического тензора. Тогда для кокасательного вектора $\xi=\left(\xi_{1}, \xi_{2}\right)$ величина $g^{i j} \xi_{i} \xi_{j}$ является инвариантом при допустимых преобразованиях координат. Для любой точки $x=\left(x^{1}, x^{2}\right)$ поворотом осей можно добиться, чтобы $z_{12}(x)=0$. Из соотношения (12), где как раз $z_{12}=0$, видно, что введенная метрика положительно определенная. Производные $z_{i j}, z_{i j k}, z_{i j k l}$ относительно допустимых преобразований координат являются координатами симметричных ковариантных тензоров соответствующих рангов. Будем поднимать и опускать индексы у тензоров с помощью введенной метрики, в частности,

$$
z_{j k}^{i}=g^{i a} z_{a j k}, \quad z^{i j k}=g^{i a} g^{j b} g^{k c} z_{a b c} .
$$

Рассмотрим тензоры

$$
P_{i j k l}=z_{i l}^{a} z_{a j k}-z_{i k}^{a} z_{a j l}, \quad P_{i k}=z_{h i}^{j} z_{j k}^{h}
$$

и инвариант $P=z_{i j k} z^{i j k}$. Очевидно, $P \geqslant 0$. Причем, если $P(x)=0$ в точке $x=\left(x^{1}, x^{2}\right)$, то все третьи производные $z_{i j k}(x)$ обращаются в нуль. 
Пусть $\Delta$ - оператор Лапласа-Бельтрами относительно введенной метрики, ковариантные производные будем обозначать с помощю запятой. Сначала получим некоторую инвариантную оценку для $\Delta(\sqrt{P})$ в произвольной точке $x=\left(x^{1}, x^{2}\right)$, где $P(x) \neq 0$.

Имеем (см. [2])

$$
\begin{aligned}
\Delta(\sqrt{P}) & =\frac{\Delta P}{2 P^{1 / 2}}-\frac{g^{i j} P_{, i} P_{, j}}{4 P^{3 / 2}}, \\
\frac{1}{2} \Delta P & =z^{i j k} \Delta z_{i j k}+z^{i j k, l} z_{i j k, l} .
\end{aligned}
$$

Обозначив $B_{a b c i j k l}=\frac{1}{2}\left(z_{a b c, i} z_{j k l}-z_{a b c} z_{j k l, i}\right)$, получим

$$
\frac{2 B_{a b c i j k l} B^{a b c i j k l}}{P^{3 / 2}}=\frac{z_{a b c, i} z^{a b c, i}}{P^{1 / 2}}-\frac{g^{i j} P_{, i} P_{, j}}{4 P^{3 / 2}} \geqslant 0 .
$$

Из соотношений (19), (20) и последнего неравенства следует

$$
\Delta(\sqrt{P}) \geqslant \frac{z^{i j k} \Delta z_{i j k}}{P^{1 / 2}}
$$

Дифференцируя уравнение $\operatorname{det}\left(z_{i j}\right)-\varphi\left(c_{11} z_{22}+2 c_{12} z_{12}+c_{22} z_{11}\right)=0$ по $x^{k}$, получим

$$
z_{i j k} g^{i j}=0
$$

Следовательно, $g^{l q} z_{i q l, j k}=0$. Тогда

$\Delta z_{i j k}=g^{l q} z_{i j k, l q}=g^{l q}\left(z_{i j l, k q}-z_{i j l, q k}\right)+g^{l q}\left(z_{i j l, q}-z_{i q l, j}\right)_{, k}+g^{l q}\left(z_{i j k, l}-z_{i j l, k}\right)_{, q}$.

Вычисляя, имеем

$$
\begin{aligned}
z^{i j k} \Delta z_{i j k}= & z^{i j k} g^{l q}\left(-5 z_{i j h} \Gamma_{q p}^{h} \Gamma_{k l}^{p}+6 z_{i h l} \Gamma_{k p}^{h} \Gamma_{q j}^{p}-z_{i j h} \Gamma_{k p}^{h} \Gamma_{q l}^{p}\right. \\
& -2 z_{h j l} \Gamma_{q p}^{h} \Gamma_{k i}^{p}+4 z_{h q p} \Gamma_{i j}^{h} \Gamma_{l k}^{p}-4 z_{p h k} \Gamma_{j l}^{h} \Gamma_{i q}^{p}-2 z_{h j l k} \Gamma_{i q}^{h}-z_{i j h k} \Gamma_{l q}^{h} \\
& +3 z_{h q l k} \Gamma_{i j}^{h}-3 z_{j k}^{h}\left(\frac{1}{2} \partial_{l q} g_{i h}-\Gamma_{i l}^{a} \partial_{q} g_{a h}\right) \\
& \left.+2 z_{j l}^{h}\left(\frac{1}{2} \partial_{i k} g_{q h}-\Gamma_{q i}^{a} \partial_{k} g_{a h}\right)\right)
\end{aligned}
$$

Здесь $\Gamma_{i j}^{k}$ - символы Кристоффеля для введенной метрики.

Сделаем унимодулярную аффинную замену координат $x^{1}, x^{2}$, чтобы стало $c_{11}=c_{22}=1, c_{12}=0$ и $z_{12}=0$ в точке $x$. Тогда в этой точке получим $g^{11}=z_{22}-\varphi^{\prime}, g^{22}=z_{11}-\varphi^{\prime}, g^{12}=0$.

Следовательно, в силу диагональности метрики в точке $x$ для всех $i, j, k$

$$
P \geqslant z_{i j k}^{2} g^{i i} g^{j j} g^{k k}, \quad P \geqslant\left(z_{i j}^{k}\right)^{2} g^{i i} g^{j j} g_{k k}, \quad P \geqslant\left(z^{i j k}\right)^{2} g_{i i} g_{j j} g_{k k}
$$


(без суммирования). Поэтому

$$
\left|z_{i j k}\right| \leqslant\left(\frac{P}{g^{i i} g^{j j} g^{k k}}\right)^{1 / 2}, \quad\left|z_{i j}^{k}\right| \leqslant\left(\frac{P}{g^{i i} g^{j j} g_{k k}}\right)^{1 / 2}, \quad\left|z^{i j k}\right| \leqslant\left(\frac{P}{g_{i i} g_{j j} g_{k k}}\right)^{1 / 2} .
$$

Так как на всей плоскости

$$
\partial_{p} g_{i i}=\frac{z_{i i p}}{D}+\frac{\left(z_{11 p}+z_{22 p}\right)\left(z_{i i}-\varphi^{\prime}+z_{12}^{2}\right) \varphi^{\prime \prime}}{D^{2}}
$$

где $D=\operatorname{det}\left(g^{i j}\right)$, то используя (22), получаем в точке $x$

$$
\partial_{p} g_{i i}=\frac{z_{i i p}}{D}+\varphi^{\prime \prime} \frac{z_{i i p}\left(z_{i i}-z_{j j}\right)}{D\left(z_{j j}-\varphi^{\prime}\right)}
$$

где $j=i+1(\bmod 2)$. А так как в силу $(5)\left|\varphi^{\prime \prime}\right| \leqslant \varepsilon \varphi /\left(z_{11}+z_{22}\right)^{2}$, то, учитывая, что $\left|z_{j j}-\varphi^{\prime}\right| \geqslant(1-\varepsilon) z_{j j}, D \geqslant(1-\varepsilon)^{3}$, запишем

$$
\partial_{p} g_{i i}=\frac{z_{i i p}}{D}+\varepsilon_{i i p} z_{i i p}
$$

где $\varepsilon_{i i p}$ не зависит от $p$ и $\left|\varepsilon_{i i p}\right| \leqslant \varepsilon /(1-\varepsilon)^{4}$.

Суммирование по повторяющимся индексам будем проводить только в случае, когда один из них верхний, а другой нижний. Если вверху или внизу стоит несколько одинаковых индексов, то при суммировании они рассматриваются как один, например, $g^{q q} z_{q q h} \varepsilon_{q h q}=\sum_{q} g^{q q} z_{q q h} \varepsilon_{q h q}$. В точке $x$ получаем также $\partial_{p} g_{12}=$ $z_{12 p} / D$, для удобства запишем это в виде

$$
\partial_{p} g_{12}=\frac{z_{12 p}}{D}+\varepsilon_{12 p} z_{12 p}
$$

где $\varepsilon_{12 p}=0$.

Дифференцируя (22) по $x^{r}$ в точке $x$, получаем

$$
g^{i i} z_{i i k r}=-z_{i j k} \partial_{r} g^{i j}
$$

а так как

$$
\begin{aligned}
\left|\partial_{r} g^{11}\right| & =\left|z_{22 r}-\left(z_{22 r}+z_{11 r}\right) \varphi^{\prime \prime}\right|=\left|z_{22 r}\right|\left|1-\varphi^{\prime \prime} \frac{z_{22}-z_{11}}{z_{22}-\varphi^{\prime}}\right| \\
& \leqslant \frac{\left|z_{22 r}\right|}{1-\varepsilon} \leqslant\left(\frac{P}{g^{22} g^{22} g^{r r}}\right)^{1 / 2} \frac{1}{1-\varepsilon}
\end{aligned}
$$

и, аналогично,

$$
\left|\partial_{r} g^{22}\right| \leqslant\left(\frac{P}{g^{11} g^{11} g^{r r}}\right)^{1 / 2} \frac{1}{1-\varepsilon}, \quad\left|\partial_{r} g^{12}\right|=\left|z_{12 r}\right| \leqslant\left(\frac{P}{g^{11} g^{22} g^{r r}}\right)^{1 / 2},
$$

TO

$$
\left|g^{i i} z_{i i k r}\right| \leqslant \frac{4 P}{(1-\varepsilon) D\left(g^{k k} g^{r r}\right)^{1 / 2}}
$$


Для вторых производных метрики в точке $x$ с учетом (27)-(30) и (6) получаем

$$
\partial_{p q} g_{i j}=\frac{z_{i j p q}}{D}+\varepsilon_{i j p q} z_{i j p q}+\bar{\varepsilon}_{i j p q}
$$

где $\varepsilon_{i j p q}=\varepsilon_{i j p}$, т.е. те же, что в $(27),(28)$, а

$$
\left|\bar{\varepsilon}_{i j p q}\right| \leqslant \frac{10(1+\varepsilon)^{2} \varepsilon P}{\left(g^{i i} g^{j j} g^{p p} g^{q q}\right)^{1 / 2}(1-\varepsilon)^{9}} .
$$

Из (22) и симметрии $z^{i j k}$ и $g^{l q}$ следует

$$
z^{i j k} g^{l q}\left(-z_{h j l k} z_{i q}^{h}-\frac{1}{2} z_{i j h k} z_{l q}^{h}+\frac{3}{2} z_{h q l k} z_{i j}^{h}-\frac{3}{2} z_{j k}^{h} z_{l q i h}+z_{j l}^{h} z_{q h i k}\right)=0 .
$$

Отсюда и из (27)-(31) получаем

$$
\begin{aligned}
z^{i j k} g^{l q}\left(-2 z_{h j l k} \Gamma_{i q}^{h}-z_{i j h k} \Gamma_{l q}^{h}+3 z_{h q l k} \Gamma_{i j}^{h}-\frac{3}{2} z_{j k}^{h} \partial_{l q} g_{i h}+z_{j l}^{h} \partial_{i k} g_{q h}\right) \\
=z^{i j k} g^{q q} g^{h h}\left(-\frac{1}{2} z_{i j k h} z_{q q h}\left(2 \varepsilon_{q h q}-\varepsilon_{q q h}\right)\right. \\
\left.\quad+\frac{3}{2} z_{q q h k} z_{i j h}\left(2 \varepsilon_{i h j}-\varepsilon_{i j h}-\varepsilon_{k h q q}\right)-\frac{3}{2} z_{h j k} \bar{\varepsilon}_{i h q q}+z_{h j q} \bar{\varepsilon}_{q h i k}\right) .
\end{aligned}
$$

Далее, так как $P_{, h}=2 z^{i j k} z_{i j k, h}=2 z^{i j k}\left(z_{i j k h}-3 z_{p j k} \Gamma_{i h}^{p}\right)$, то

$$
z^{i j k} z_{i j k h}=\frac{1}{2} P_{, h}+3 z_{p j k} \Gamma_{i h}^{p} z^{i j k}
$$

Используя неравенство Коши для каждого слагаемого, получаем

$$
\left|P_{, h} g^{q q} g^{h h} z_{q q h}\left(2 \varepsilon_{q h q}-\varepsilon_{q q h}\right)\right| \leqslant \frac{2 P_{, h}^{2} g^{h h}}{\varepsilon_{1} P}+\frac{4 P \varepsilon^{2} \varepsilon_{1}}{(1-\varepsilon)^{8}} .
$$

Здесь $\varepsilon_{1}$ может быть любым положительным числом.

В силу диагональности метрики в точке $x$

$$
|\operatorname{grad} \sqrt{P}|^{2}=\frac{g^{h h} P_{, h}^{2}}{4 P} .
$$

Учитывая, что $\varepsilon_{k h q q}$ одинаковы для всех $q$, из (30) получаем, используя $(24)$ и оценки для $\varepsilon_{i j k}$ и $\varepsilon_{i j k l}$, что

$$
\left|z^{i j k} g^{q q} g^{h h} z_{q q h k} z_{i j h}\left(2 \varepsilon_{i h j}-\varepsilon_{i j h}-\varepsilon_{k h q q}\right)\right| \leqslant \frac{128 \varepsilon P^{2}}{(1-\varepsilon)^{8}} .
$$

Соотношения (32)-(36) позволяют оценить слагаемые в (23), содержащие четвертые производные функции $z\left(x^{1}, x^{2}\right)$ :

$$
\begin{aligned}
& \left|z^{i j k} g^{l q}\left(-2 z_{h j l k} \Gamma_{i q}^{h}-z_{i j h k} \Gamma_{l q}^{h}+3 z_{h q l k} \Gamma_{i j}^{h}-\frac{3}{2} z_{j k}^{h} \partial_{l q} g_{i h}+z_{j l}^{h} \partial_{i k} g_{q h}\right)\right| \\
& \quad \leqslant \frac{2|\operatorname{grad} \sqrt{P}|^{2}}{\varepsilon_{1}}+\left(\frac{144}{(1-\varepsilon)^{4}}+\frac{128}{(1-\varepsilon)^{8}}+\frac{800(1+\varepsilon)^{2}}{(1-\varepsilon)^{9}}+\frac{4 \varepsilon \varepsilon_{1}}{(1-\varepsilon)^{8}}\right) P^{2} \varepsilon .
\end{aligned}
$$


Подставив вместо символов Кристоффеля их выражение через метрику, используя неравенства $(27),(28),(24)$ и учитьвая, что

$$
\left|\varepsilon_{h p q} z_{h p q}+\varepsilon_{h q p} z_{h q p}-\varepsilon_{q p h} z_{q p h}\right| \leqslant \frac{\varepsilon P^{1 / 2}}{(1-\varepsilon)^{4}\left(g^{h h} g^{p p} g^{q q}\right)^{1 / 2}}
$$

для любых $h, p, q=1,2$, получаем оценку

$$
\begin{aligned}
& z^{i j k} g^{l q}\left(-5 z_{i j h} \Gamma_{q p}^{h} \Gamma_{k l}^{p}+6 z_{i h l} \Gamma_{k p}^{h} \Gamma_{q j}^{p}-z_{i j h} \Gamma_{k p}^{h} \Gamma_{q l}^{p}-2 z_{h j l} \Gamma_{q p}^{h} \Gamma_{k i}^{p}\right. \\
&\left.\quad+4 z_{h q p} \Gamma_{i j}^{h} \Gamma_{l k}^{p}-4 z_{p h k} \Gamma_{j l}^{h} \Gamma_{i q}^{p}+3 z_{j k}^{h} \Gamma_{i l}^{a} \partial_{q} g_{a h}-2 z_{j l}^{h} \Gamma_{q i}^{a} \partial_{k} g_{a h}\right) \\
& \geqslant \frac{1}{4 D^{2}}\left(3 z^{i j k} z_{i j}^{s} z_{l s q} z_{k}^{l q}-2 z^{i j k} z_{l}^{s q} z_{l s k} z_{q j}^{l}\right)-2^{6}\left(\frac{16 \varepsilon}{(1-\varepsilon)^{4}}+\frac{8 \varepsilon^{2}}{(1-\varepsilon)^{8}}\right) P^{2}
\end{aligned}
$$

Хотя тензоры $P_{i j k l}, P_{i k}$ у нас не имеют непосредственного геометрического смысла, для них выполняются все алгебраические свойства соответствующих тензоров из [2]. В частности,

$$
\begin{aligned}
& g^{i k} P_{i k}=P, \quad P_{i k} P^{i k} \geqslant \frac{1}{2} P^{2}, \quad P_{i j k l} P^{i j k l}=2 P_{i j} P^{i j} \\
& P_{i j k l} P^{i j k l}+P_{i k} P^{i k}=3 z^{i j k} z_{i j}^{s} z_{l s q} z_{k}^{l q}-2 z^{i j k} z_{l}^{s q} z_{l s k} z_{q j}^{l} .
\end{aligned}
$$

Следовательно,

$$
3 z^{i j k} z_{i j}^{s} z_{l s q} z_{k}^{l q}-2 z^{i j k} z_{l}^{s q} z_{l s k} z_{q j}^{l} \geqslant \frac{3}{2} P^{2} .
$$

Для краткости введем обозначение

$$
A=\frac{3}{8(1+\varepsilon)^{6}}-\varepsilon\left(\frac{1168}{(1-\varepsilon)^{4}}+\frac{800(1+\varepsilon)^{2}}{(1-\varepsilon)^{9}}+\frac{128+4 \varepsilon \varepsilon_{1}+512 \varepsilon}{(1-\varepsilon)^{8}}\right) .
$$

Из (21), (23), (37)-(39), учитывая, что $D \leqslant(1+\varepsilon)^{3}$, получаем

$$
\Delta(\sqrt{P}) \geqslant A P^{3 / 2}-\frac{2|\operatorname{grad} \sqrt{P}|^{2}}{\varepsilon_{1} \sqrt{P}} .
$$

Эта оценка выполняется в любой точке $x=\left(x^{1}, x^{2}\right)$, где $P(x) \neq 0$, с любым $\varepsilon_{1}>0$ и является инвариантной относительно допустимых преобразований координат $x^{1}, x^{2}$.

\section{§5. Доказательство теоремы об уравнении для несобственных выпуклых аффинных сфер}

Пусть в прямоугольной системе координат параболоид П удовлетворяет условию $\operatorname{det}\left(c_{i j}\right)=1$ и для поверхностей П и $\Phi$ вьполняется уравнение $(7)$. Тогда из результатов $\S 3$ следует, что для вторых производных $z_{i i}$ решения уравнения (7) существуют положительные абсолютные оценки сверху и снизу, зависящие только от $\varepsilon$ и $d_{2}\left(\right.$ при $\left.(1-\varepsilon)^{3}>4 \varepsilon\right)$.

Отсюда следует, что метрическая топология на плоскости $z=0$ относительно введенной метрики эквивалентна стандартной эвклидовой. 
Рассмотрим на положительной полуоси $t$ обыкновенное дифференциальное уравнение

$$
y^{\prime \prime}+\frac{B}{t} y^{\prime}+\frac{y^{\prime 2}}{\varepsilon_{0} y}=c_{0} y^{3}
$$

с начальњыми условиями

$$
y(0)=a, \quad y^{\prime}(0)=0,
$$

где $B>0, \varepsilon_{0}>0, c_{0}>0, a>0$ - некоторые константы.

В работе [6] доказано, что если $y(t)$ - решение уравнения (42) для $t \geqslant 0$ с начальными условиями (43), то сушествует такое число $d>0$, зависящее от $a, B$, $\varepsilon_{0}, c_{0}$, что $\lim _{t \rightarrow d} y(t)=+\infty$. Кроме того, $y^{\prime}(t)>0$ при $t \in(0, d)$.

Предположим, что в некоторой точке значение инварианта $P$ не равно нулю. Чтобы не вводить новых обозначений, обозначим эту точку за $O$ и положим $\sqrt{P(O)}=2 a$. Предположим, что нам удалось найти положительную функцию $v\left(x^{1}, x^{2}\right)$ обладающую следующими свойствами:

1) функция $v$ определена в области $\Sigma$ такой, что $\Sigma$ содержит точку $O$ и множество $\bar{\Sigma}$ компактно;

2) $v(O)=a$;

3) $\Delta v \leqslant(A-H) v^{3}+H P^{3 / 2}-4|\operatorname{grad} v|^{2} /\left(\varepsilon_{1} v\right)+2|\operatorname{grad} v|^{2} /\left(\varepsilon_{1} \sqrt{P}\right)$, где $A, \varepsilon_{1}$ те же, что в неравенстве (41), и положительные числа $\varepsilon_{1}$ и $H$ таковы, что $A-H>0$; если $P(x)=0$, то считаем, что правая часть последнего дифференциального неравенства принимает значение $+\infty$;

4) $v(x) \rightarrow+\infty$ при $x \rightarrow \partial \Sigma$.

Тогда функция $\sqrt{P}-v$ достигает максимума в точке $\widetilde{x} \in \Sigma$, где $\operatorname{grad}(\sqrt{P}-v)=0$; это означает, что $\operatorname{grad} \sqrt{P}(\widetilde{x})=\operatorname{grad} v(\widetilde{x})$. Кроме того, в точке $\widetilde{x}$ имеем $\sqrt{P(\widetilde{x})}-v(\widetilde{x}) \geqslant a$, следовательно, $\sqrt{P(\widetilde{x})}>v(\widetilde{x})>0$. Отсюда в точке $\widetilde{x}$ будем иметь

$$
\Delta v \leqslant(A-H) v^{3}+H P^{3 / 2}-\frac{2|\operatorname{grad} \sqrt{P}|^{2}}{\varepsilon_{1} \sqrt{P}} .
$$

Вьчитая из неравенства (41) неравенство (44), получаем в точке $\widetilde{x}$

$$
\Delta(\sqrt{P}-v) \geqslant(A-H)\left(P^{3 / 2}-v^{3}\right)>0 .
$$

Противоречие с тем, что в этой точке достигается максимум функции $\sqrt{P(x)}-v(x)$. Следовательно, $P(x) \equiv 0$ и все третьи производные функции $z\left(x^{1}, x^{2}\right)$ тоже тождественно равны нулю. Значит, $z\left(x^{1}, x^{2}\right)$ - квадратичный полином.

В тех точках, где $P(x)=0$, функция $\sqrt{P(x)}$ не принадлежит классу $C^{2}$. Но в этих точках функция $\sqrt{P}-v$ не может достигать максимума, и предыдущие рассуждения являются законными.

Приступим к построению функции $v$ со свойствами 1)-4).

Возьмем точку $O$ за начало аффинной системы координат на плоскости $z=0$. Введем “афффинное расстояние" $s(x)=\sqrt{c_{i j} x^{i} x^{j}}$ от точки $O$ до точки $x=\left(x^{1}, x^{2}\right)$. На любом луче, исходящем из точки $O, s$ изменяется от 0 до $+\infty$. Пусть $y(t)-$ решение уравнения (42) с начальными условиями (43) при $t \geqslant 0$. Тогда функция $v(x)=y(s(x))$, очевидно, обладает свойствами 1), 2), 4). Докажем, что можно так подобрать постоянные $B, \varepsilon_{0}, c_{0}$, чтобы выполнялось свойство 3$)$. Заметим, что 
функция $v(x)$ принадлежит только классу $C^{1}$, поэтому дифференциальные неравенства с участием $v(x)$ мы понимаем в расширенном смысле, как это делается в работе [7]. Там дано определение, которое в нашем случае будет иметь следующий вид.

ОПРЕДЕЛЕНИЕ. Будем Говорить, что $\Delta v($ слабо $) \leqslant u$ на множестве $\Sigma$, если для каждой точки $x_{0} \in \Sigma$ и каждого числа $\delta>0$ существуют окрестность $V_{\delta, x_{0}}$ точки $x_{0}$ и функция $v_{\delta, x_{0}}(x) \in C^{2}$ в $V_{\delta, x_{0}}$, которая удовлетворяет следующим условиям:

a) $v(x)-v_{\delta, x_{0}}(x)$ достигает максимального значения на $V_{\delta, x_{0}}$ в точке $x_{0}$;

b) $\Delta v_{\delta, x_{0}}\left(x_{0}\right)<u\left(x_{0}\right)+\delta$.

При этом (см. [7]) принцип максимума имеет место в обычной формулировке.

Всюду в области $\Sigma$ за исключением точки $O$ имеем

$$
\Delta v=g^{i j} v_{, i j}=g^{i j} \frac{\partial s}{\partial x^{i}} \frac{\partial s}{\partial x^{j}} y^{\prime \prime}+y^{\prime} \Delta s .
$$

Так как функция $s(x)$ инвариантна при замене базисных векторов аффинной системы координат на плоскости $z=0$, то можно вычислять $\Delta s(x)$ и $\left.\left(g^{i j} \partial s / \partial x^{i} \partial s / \partial x^{j}\right)\right|_{x}$ в такой аффинной системе координат, где матрица $\left(c_{i j}\right)$ единичная, а матрица $\left(z_{i j}\right)$ диагональная в точке $x$. При таком преобразовании координат вторые производные $z_{i i}$ функции $z\left(x^{1}, x^{2}\right)$ изменятся не более чем в $\lambda_{0}$ раз, где $\lambda_{0}$ - максимальное собственное значение матрицы $\left(c_{i j}\right)$. Значит, по-прежнему, $z_{i i}$ имеют абсолютные оценки сверху и снизу, зависящие от $\varepsilon$ и $d_{2}$. Обозначим их через $M_{1}$ и $M_{2}$ :

$$
0<M_{2}\left(\varepsilon, d_{2}\right) \leqslant z_{i i} \leqslant M_{1}\left(\varepsilon, d_{2}\right)
$$

Из $(22),(24),(27),(28)$ получаем (здесь $\left.x_{q}=x^{q}\right)$

$\Delta s=g^{i j} s_{, i j}=\frac{g^{11}\left(x^{2}\right)^{2}+g^{22}\left(x^{1}\right)^{2}}{s\left(\left(x^{1}\right)^{2}+\left(x^{2}\right)^{2}\right)}-g^{i i} \frac{x_{q}}{s} \Gamma_{i i}^{q} \leqslant \frac{1}{s} \max _{i} g^{i i}+\frac{2 \varepsilon P^{1 / 2}}{(1-\varepsilon)^{4}} \max _{i} \sqrt{g^{i i}}$.

А так как $g^{i i}=z_{j j}-\varphi^{\prime} \leqslant(1+\varepsilon) z_{j j}, j=i+1(\bmod 2)$, то

$$
\Delta s \leqslant \frac{(1+\varepsilon) M_{1}}{s}+\frac{2 \varepsilon\left(P(1+\varepsilon) M_{1}\right)^{1 / 2}}{(1+\varepsilon)^{4}} .
$$

Заметим, что

$$
\begin{gathered}
|\operatorname{grad} v|^{2}=y^{2} g^{i j} \frac{\partial s}{\partial x^{i}} \frac{\partial s}{\partial x^{j}} \\
g^{i j} \frac{\partial s}{\partial x^{i}} \frac{\partial s}{\partial x^{j}}=\frac{g^{11}\left(x^{1}\right)^{2}+g^{22}\left(x^{2}\right)^{2}}{\left(x^{1}\right)^{2}+\left(x^{2}\right)^{2}} .
\end{gathered}
$$

Следовательно,

$$
(1-\varepsilon) M_{2} \leqslant g^{i j} \frac{\partial s}{\partial x^{i}} \frac{\partial s}{\partial x^{j}} \leqslant(1+\varepsilon) M_{1}
$$


Теперь подставим в (45) выражение для $y^{\prime \prime}(s)$, полученное из (42), воспользуемся оценками $(48),(46)$, равенством $(47)$, учтем, что $y^{\prime}(s)>0$, и возьмем

$$
B=\frac{(1+\varepsilon) M_{1}}{(1-\varepsilon) M_{2}}, \quad c_{0}=\frac{A-H}{(1+\varepsilon) M_{1}}, \quad \varepsilon_{0}=\frac{\varepsilon_{1}}{4},
$$

где $H>0$ выберем чуть позже так, чтобы выполнялось условие $A-H>0$. Тогда получим в точке $x$

$$
\Delta v \leqslant(A-H) v^{3}-\frac{4|\operatorname{grad} v|^{2}}{\varepsilon_{1} v}+\frac{2 \varepsilon\left(P(1+\varepsilon) M_{1}\right)^{1 / 2}}{(1-\varepsilon)^{4}} y^{\prime} .
$$

Используя неравенство Коши с постоянной $\varepsilon_{1} \sqrt{P} /\left(2 M_{2}(1-\varepsilon)\right)$, получаем

$$
\frac{2 \varepsilon\left(P(1+\varepsilon) M_{1}\right)^{1 / 2} y^{\prime}}{(1-\varepsilon)^{4}} \leqslant \frac{2 y^{\prime 2}(1-\varepsilon) M_{2}}{\varepsilon_{1} \sqrt{P}}+\frac{(1+\varepsilon) \varepsilon^{2} M_{1} P^{3 / 2} \varepsilon_{1}}{2(1-\varepsilon)^{9} M_{2}} .
$$

Из (47) и (48) следует $y^{\prime 2} \leqslant|\operatorname{grad} v|^{2} /\left((1-\varepsilon) M_{2}\right)$.

Теперь введем $H$. Положим

$$
\frac{(1+\varepsilon) \varepsilon^{2} M_{1} \varepsilon_{1}}{2(1-\varepsilon)^{9} M_{2}}=H,
$$

где $\varepsilon_{1}$ еще нужно будет подобрать. Из последних двух неравенств получим

Отсюда и из (49)

$$
\frac{2 \varepsilon\left(P(1+\varepsilon) M_{1}\right)^{1 / 2}}{(1-\varepsilon)^{4}} y^{\prime} \leqslant \frac{2|\operatorname{grad} v|^{2}}{\varepsilon_{1} \sqrt{P}}+H P^{3 / 2} .
$$

$$
\Delta v \leqslant(A-H) v^{3}+H P^{3 / 2}-\frac{4|\operatorname{grad} v|^{2}}{\varepsilon_{1} v}+\frac{2|\operatorname{grad} v|^{2}}{\varepsilon_{1} \sqrt{P}} .
$$

Из определения $H$ и $A$ видно, что при

$$
\frac{3}{8(1+\varepsilon)^{6}}-\varepsilon\left(\frac{1168}{(1-\varepsilon)^{4}}+\frac{128}{(1-\varepsilon)^{8}}+\frac{800(1+\varepsilon)^{2}}{(1-\varepsilon)^{9}}+\frac{512 \varepsilon}{(1-\varepsilon)^{8}}\right)>0
$$

$\left(\varepsilon<\approx 10^{-4}\right)$ найдется такое $\varepsilon_{1}$, что $A-H>0$ и условие 3$)$ для функции $v$ выполняется во всей ее области определения за исключением, быть может, точки $O$.

Теперь проверим, что функция $v$ удовлетворяет условию 3$)$ и в точке $O$. Заметим, что в точке $O$ правая часть неравенства в 3$)$ равна $a^{3}(A+7 H)$. Поэтому берем

$$
v_{\delta, O}\left(x^{1}, x^{2}\right)=a+\frac{1}{2} \frac{a^{3}(A+7 H)+\delta}{g^{11}(O)+g^{22}(O)}\left(\left(x^{1}\right)^{2}+\left(x^{2}\right)^{2}\right) .
$$

Тогда условие b) определения выполняется. Далее, из (42) получаем $y^{\prime \prime}(0)=$ $c_{0} a^{3} /(1+B)$ и проверяем, что $c_{0} a^{3} /(1+B)<\left(a^{3}(A+7 H)+\delta\right) /\left(g^{11}+g^{22}\right)$. Теперь выполнимость условия а) определения следует из разложения Тейлора $y(s)=a+\frac{1}{2} y^{\prime \prime}(0) s^{2}+\cdots$.

Функция $v\left(x^{1}, x^{2}\right)$ построена.

В процессе построений нам потребовалась пятикратная дифференцируемость функции $z\left(x^{1}, x^{2}\right)$. По теореме 11.3 из [8] о гладкости решений эллиптических уравнений для выполнения этого условия достаточно потребовать, чтобы $\varphi(t) \in C^{3, \alpha}$.

Отметим еще, что так как $\lambda_{1}+\lambda_{2} \geqslant 2 \sqrt{\lambda_{1} \lambda_{2}}=2 \sqrt{\varphi} \geqslant 2 \sqrt{1-\varepsilon}$, то выполнимость условий (4)-(6) достаточно потребовать при $t \geqslant 2 \sqrt{1-\varepsilon}$.

Итак, доказана 
ТЕОРемА. Пусть функиия $\varphi(t)$ определена для $t>0$, имеет класс $C^{3, \alpha}$, $0<\alpha<1$, и удовлетворяет условиям

$$
\begin{aligned}
1-\varepsilon \leqslant \varphi(t) \leqslant 1+\varepsilon, & t>0, \\
\left|\varphi^{\prime}(t)\right| \leqslant \varepsilon \frac{\varphi(t)}{t}, & t \geqslant 2 \sqrt{1-\varepsilon} \\
\left|\varphi^{\prime \prime}(t)\right| \leqslant \varepsilon \frac{\varphi(t)}{t^{2}}, & t \geqslant 2 \sqrt{1-\varepsilon} \\
\left|\varphi^{\prime \prime \prime}(t)\right| \leqslant \varepsilon \frac{\varphi(t)}{t^{3}}, & t \geqslant 2 \sqrt{1-\varepsilon}
\end{aligned}
$$

с положительным $\varepsilon$, для которого выполняется неравенство (50). Тогда всякая полная поверхность, заданная в прямоугольной системе координат в виде $z=z\left(x^{1}, x^{2}\right)$, где функиия $z\left(x^{1}, x^{2}\right)$ удовлетворяет уравнению

$$
\operatorname{det}\left(z_{i j}\right)=\varphi\left(z_{11}+z_{22}\right)
$$

является әллиптическим параболоидом.

Условиям теоремы удовлетворяет, например, функция

$$
\varphi(t)=1+\frac{\varepsilon}{14} \sin \frac{1}{t}, \quad t>0
$$

\section{Список литературы}

1. Jörgens $K$. Über die Lösungen der Differentialgleichung $r t-s^{2}=1 / /$ Math. Ann. 1954 . V. 127. P. 130-134.

2. Calabi E. Improper affine hyperspheres of convex type and a generalizations of a theorem by K. Jörgens // Michigan Math. J. 1958. V. 5. № 2. P. 105-126.

3. Погорелов А. В. Многомерная проблема Минковского. М.: Наука, 1975.

4. Caffarelli L., Nirenberg L., Spruck J. The Dirichlet problem for nonlinear second order elliptic equations,III: Functions of the eigenvalues of the Hessian // Acta Math. 1985. V. 155. № 3, 4. P. 261-301.

5. Погорелов А.В. Многомерное уравнение Монжа-Ампера $\operatorname{det}\left(z_{i j}\right)=\varphi\left(z_{1}, \ldots, z_{n}, z\right.$, $\left.x_{1}, \ldots, x_{n}\right)$. М.: Наука, 1988.

6. Кокарев B. Н. О полных выпуклых решениях уравнения $\operatorname{spur}_{m}\left(z_{i j}\right)=1 / /$ Матем. физика, анализ, геом. 1996. Т. 3. №1/2. С. 102-117.

7. Calabi E. An extension of E. Hopf's maximum principle with an application to Riemannian geometry // Duke Match. J. 1958. V. 25. P. 45-56.

8. Агмон C., Дуглис A., Ниренберг Л. Оценки вблизи границы решений эллиптических уравнений в частных производных при общих граничных условиях.1. М.: ИЛ, 1962.

Самарский государственньй университет

E-mail: kokarev@ssu.samara.ru
Поступила в редакцию 12.11.2001 и 26.08.2002 